\title{
Quantitative Analysis of Adulterations in Oat Flour by FT-NIR Spectroscopy, Incomplete Unbalanced Randomized Block Design, and Partial Least Squares
}

\author{
Ning Wang, Xingxiang Zhang, Zhuo Yu, Guodong Li, and Bin Zhou \\ School of Material Science and Engineering, Tianjin Municipal Key Lab of Fiber Modification and Functional Fiber, \\ Tianjin Polytechnic University, Tianjin 300389, China
}

Correspondence should be addressed to Xingxiang Zhang; 1536779197@qq.com

Received 19 April 2014; Accepted 21 June 2014; Published 20 July 2014

Academic Editor: Constantinos Georgiou

Copyright (C) 2014 Ning Wang et al. This is an open access article distributed under the Creative Commons Attribution License, which permits unrestricted use, distribution, and reproduction in any medium, provided the original work is properly cited.

\begin{abstract}
This paper developed a rapid and nondestructive method for quantitative analysis of a cheaper adulterant (wheat flour) in oat flour by NIR spectroscopy and chemometrics. Reflectance FT-NIR spectra in the range of 4000 to $12000 \mathrm{~cm}^{-1}$ of 300 oat flour objects adulterated with wheat flour were measured. The doping levels of wheat flour ranged from $5 \%$ to $50 \%$ (w/w). To ensure the generalization performance of the method, both the oat and the wheat flour samples were collected from different producing areas and an incomplete unbalanced randomized block (IURB) design was performed to include the significant variations that may be encountered in future samples. Partial least squares regression (PLSR) was used to develop calibration models for predicting the levels of wheat flour. Different preprocessing methods including smoothing, taking second-order derivative (D2), and standard normal variate (SNV) transformation were investigated to improve the model accuracy of PLS. The root mean squared error of Monte Carlo cross-validation (RMSEMCCV) and root mean squared error of prediction (RMSEP) were 1.921 and 1.975 (\%, w/w) by D2-PLS, respectively. The results indicate that NIR and chemometrics can provide a rapid method for quantitative analysis of wheat flour in oat flour.
\end{abstract}

\section{Introduction}

Food adulteration and fraud have been a common problem in food production since ancient times. Food adulteration is economically motivated and is performed by the addition, substitution, or removal of food ingredients, for example, replacing or diluting high-cost ingredients with cheaper ones $[1,2]$. It is an issue that concerns not only consumers, but also food producers, sellers, regulatory agencies, and even the entire food industry chain. For consumers, food adulterations have caused growing concern about health risks, as well as the food quality and nutrition value. A notorious and common phenomenon is the adulterations of raw food materials, which can not only influence the quality of raw materials but also cause potential crisis in further processed foods $[3,4]$.

Oat is widely utilized for human consumption and food industrial uses. Due to its high nutritional value and characteristic flavor, oat flour plays an important role in the breakfast cereals group and other processed foods as an alternative or supplement to the ordinary wheat flour [5]. As a nonstaple cereal, the yield of oat is much less than wheat, so oat flour is more expensive than wheat flour. For producers and sellers, it is economically profitable to add wheat flour to oat flour. Because the appearances and physical and chemical properties of wheat and oat flours are very similar, rapid and effective methods are required to analyze the adulterations.

For food analysis and quality control, NIR spectroscopy has demonstrated some advantages, including less sample treatment, reduced analysis time and cost, and the feasibility for nondestructive analysis and online analysis. NIR spectroscopy has been widely used for analysis of grains and cereals. Hurburgh et al. [6] combined NIR spectroscopy and principal component analysis to discriminate transgenic grains and nontransgenic grains. Munck et al. [7] applied the 
NIRS technology to distinguish barley flour with different levels of lysine amino acids. NIR was also successfully used to distinguish corn samples of different genotypes $[8,9]$. For quantitative analysis, NIR technology has provided a rapid tool for analysis of different constituents or quality parameters in grain products, including corn dry-milling quality [10], protein content in wheat kernels [11], the ratio of starch amylose content to total grain in corn [12], undried rough rice constituent content [13], kernel rots and mycotoxins in maize [14], and protein, moisture, dry mass, hardness, and other residues of wheat [15] and so on [16].

Considering the large number of samples in market shelf and small private retailers, NIR is a convenient and economic technique for analysis of adulterations in oat flour. This paper was aimed at developing a rapid method for analysis of potential wheat flour added to oat flour using NIR spectrometry and chemometrics. Considering the composition variations of oats from different producing areas, an incomplete unbalanced randomized block design [17] was performed to ensure the generalization performance of multivariate calibration models.

\section{Materials and Methods}

2.1. Collection and Preparation of Samples. Pure oat flour and wheat flour objects were made from intact grains. A set of oat and wheat kernels harvested in 2013 were collected from domestic markets. Oat kernels were produced in Hebei (15), Henan (17), Gansu (17), Shanxi (18), and Qinghai (13). Wheat kernels were produced in Henan (11), Hebei (10), Jiangsu (8), Anhui (10), Shandong (11), Shanxi (7), and Heilongjiang (8). The kernels were dried in the sun and milled by a crusher. All the particles were filtered through a 200-mesh sieve.

Adulterated oat flour samples were made by mixing the oat flour with different levels of the wheat flour. The doping levels were $5 \%, 10 \%, 15 \%, 20 \%, 25 \%, 30 \%, 35 \%, 40 \%, 45 \%$, and $50 \%(\mathrm{w} / \mathrm{w})$. In order to obtain a representative nut not too large sample set, an incomplete and unbalanced randomized design [17] was performed. Because this paper was focused on quantitative analysis of wheat flour in oat flour, the producing areas of oat and wheat were considered to be two blocking factors. In this way, 120 adulterated objects were prepared for developing calibration models with 10 doping levels each having 12 objects. Another 100 adulterated objects (10 doping levels each having 10 objects) were prepared for model validation by mixing oat and wheat flour objects that were different from those used for preparation of training samples.

The NIR diffuse reflectance spectra of impacted powders were measured on a Bruker-TENSOR37 FTIR spectrometer (Bruker Optics, Ettlingen, Germany). The working range of spectrometer was $4000-12000 \mathrm{~cm}^{-1}$. The spectra were measured using a $\mathrm{PbS}$ detector with an internal gold background as the reference. The instrument resolution was $4 \mathrm{~cm}^{-1}$ and the scanning interval was $1.929 \mathrm{~cm}^{-1}$, so each spectrum contained 4148 wavelengths. Each spectrum was the average of 64 scans. For each object, three spectra were measured by stirring the powder and the average spectrum was taken.

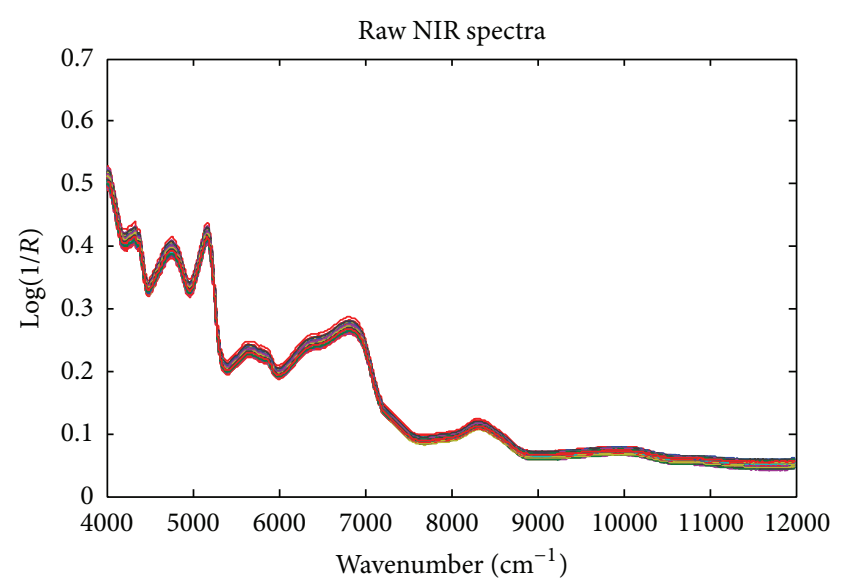

FIGURE 1: The raw NIR spectra of 120 adulterated oat flour objects with doping levels ranging from $5 \%$ to $50 \%(\mathrm{w} / \mathrm{w})$.

2.2. Data Preprocessing and Multivariate Calibration. All the data preprocessing and chemometrics models were performed using MATLAB 7.0.1 (Mathworks, Sherborn, MA). Smoothing was used to remove random noise in the data and improve the signal-to-noise ratio (SNR). In this work the S-G polynomial fitting algorithm [18] was used for smoothing for its simplicity and effectiveness. Taking secondorder derivative (D2) of spectra was performed to enhance spectral resolution and remove linear baseline shifts. The D2 spectra were also computed by S-G polynomial fitting algorithm because this method can avoid degradation of SNR compared with direct differencing. Standard normal variate (SNV) [19] transformation was performed to reduce the spectral variations caused by scattering and uneven sizes of particle.

Partial least squares (PLS) models were developed using the raw and preprocessed spectra. An important problem when performing PLS is the overfitting of models. In this paper, Monte Carlo cross-validation (MCCV) [20] was used to select the number of PLS components. MCCV can avoid the risk of overfitting by multiple random splitting of the training objects and having a higher percent of leave-out objects for prediction.

\section{Results and Discussion}

The raw NIR spectra of 120 adulterated oat flour objects are shown in Figure 1. Some of the absorbance peaks can be assigned as follows [21]: (1) the peak at $4318 \mathrm{~cm}^{-1}$ caused by the combination absorbance of $-\mathrm{CH}_{2}$ deformation and various $\mathrm{C}-\mathrm{H}$ stretching; (2) the wide peak at $4748 \mathrm{~cm}^{-1}$, overlapping of combination of $\mathrm{C}=\mathrm{O}$ stretching and peptide group deformation and combination of $\mathrm{N}-\mathrm{H}$ stretching and peptide group deformation; (3) the peak at $5167 \mathrm{~cm}^{-1}$, combination of $\mathrm{O}-\mathrm{H}$ stretching and $\mathrm{O}-\mathrm{H}$ deformation; (4) $5629 \mathrm{~cm}^{-1}$, the first overtone of symmetrical and asymmetrical $\mathrm{C}-\mathrm{H}$ stretching in $-\mathrm{CH}_{2} ;(5)$ the wide peak at $6802 \mathrm{~cm}^{-1}$, overlapping of the first overtone of $\mathrm{O}-\mathrm{H}$ stretching $\left(\sim 6900 \mathrm{~cm}^{-1}\right)$ and the first overtone of N-H stretching $\left(\sim 6500 \mathrm{~cm}^{-1}\right)$; (6) 

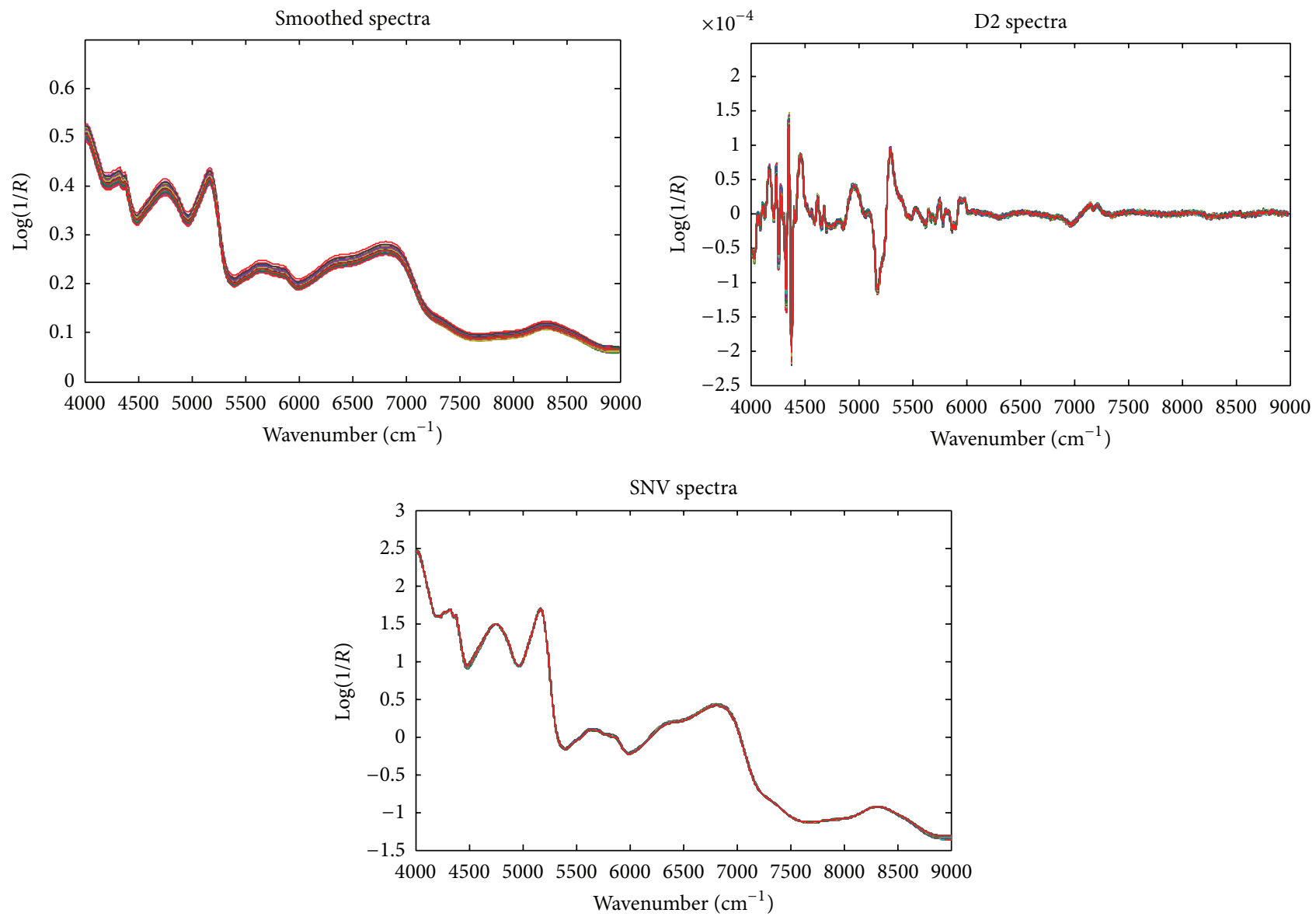

FIGURE 2: The smoothed, second-order derivative (D2) and standard normal variate (SNV) NIR spectra of 120 adulterated oat flour objects with doping levels ranging from $5 \%$ to $50 \%(\mathrm{w} / \mathrm{w})$.

$8329 \mathrm{~cm}^{-1}$, the second overtones of $\mathrm{C}-\mathrm{H}$ stretching in various groups; and (7) $9970 \mathrm{~cm}^{-1}$, the second overtones of $\mathrm{N}-\mathrm{H}$ stretching or the third overtones of $\mathrm{C}-\mathrm{H}$ stretching. The spectral interval $9000-12,000 \mathrm{~cm}^{-1}$ has no significant peaks, so this interval was not used for developing calibration models.

Smoothed, D2, and SNV spectra were shown in Figure 2. Seen from Figure 2, the D2 spectra can remove most of the backgrounds and the peak resolution was largely improved by taking D2 spectra. D2 spectra also obtained much detailed and high-frequency information. SNV transformation can remove most unwanted variations. Multivariate calibration models were developed with PLS to predict the levels of wheat flour. The number of PLS components was estimated using Ftest of MCCV. In this work, random splitting of the training set was performed for 100 times and each time $70 \%$ of the training objects were used for developing a PLS model and $30 \%$ for prediction. The pooled predicted residual sum of squares (PRESS) was computed using different numbers of PLS components. Finally, F-test was performed to select the fewest PLS components with a PRESS value not significantly higher than the minimum PRESS value. As recommended by the original literature, the significance level of the $F$-test was set to be $0.25[22,23]$.

Based on differently preprocessed spectra (9000$4000 \mathrm{~cm}^{-1}$ ) the calibration and prediction results of PLS were demonstrated in Table 1 . Seen from Table 1 , the model complexity of D2-PLS and SNV-PLS was reduced by one component compared with the PLS model using raw data. Moreover, seen from the model complexity of PLS models ( 3 or 4 components), the mixtures of wheat flour and oat flour investigated in this work were not a simple two-component system and spectral variations caused by different geographical origins had made it necessary to perform multivariate calibration. The root mean squared error of MCCV (RMSEMCCV) value was slightly reduced by smoothing (2.274), taking D2 (1.921), and SNV transformation (1.981) compared with PLS with raw data (2.388). For prediction, the lowest root mean squared error of prediction (RMSEP) of 1.975 was obtained by D2-PLS model. The training and prediction results by D2-PLS are demonstrated in Figure 3. For all of the PLS models, the differences between RMSEP and RMSEMCCV values were insignificant, indicating that both the training and test sets 

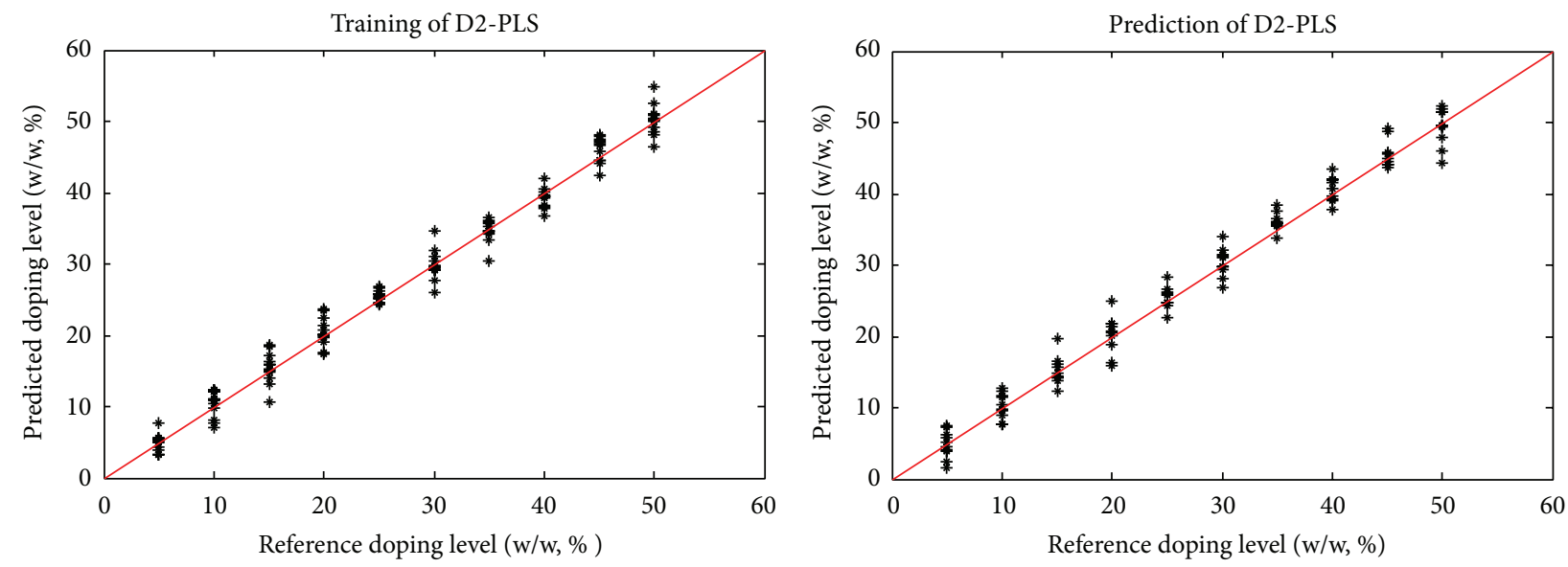

FIGURE 3: The training and prediction of contents of wheat flour in oat flour by three-component D2-PLS.

TABLE 1: Quantitative analysis of wheat flour in oat flour by PLS.

\begin{tabular}{lcccc}
\hline Preprocessing & $N^{\mathrm{a}}$ & RMSEMCCV (\%) & RMSEC (\%) & RMSEP (\%) \\
\hline Raw data & 4 & 2.388 & 2.115 & 2.213 \\
Smoothing & 4 & 2.274 & 2.102 & 2.244 \\
D2 & 3 & 1.921 & 1.781 & 1.975 \\
SNV & 3 & 1.981 & 1.842 & 2.054 \\
\hline
\end{tabular}

${ }^{\mathrm{a}}$ The number of significant PLS components.

obtained by IURB design were representative to include composition variations caused by different origins of oat and wheat flour.

\section{Conclusions}

Multivariate calibration models were developed by PLS for analysis of wheat flour in oat flour from different geographical origins. The results demonstrated that a three- or fourcomponent PLS model can accurately predict the levels of wheat flour in oat flour. Moreover, IURB design was shown to be useful to obtain representative training and test sets to include the composition variations caused by different producing areas. The developed PLS models will have a good generalization performance and are useful for quantitative analysis of oat flour in domestic market.

\section{Conflict of Interests}

Ning Wang, Xingxiang Zhang, and Bin Zhou declare no conflict of interests regarding the publication of this paper.

\section{Acknowledgment}

The authors thank Science Funds of Aviation (201329Q2001) for the financial support.

\section{References}

[1] J. H. Kalivas, C. A. Georgiou, M. Moira, I. Tsafaras, E. A. Petrakis, and G. A. Mousdis, "Food adulteration analysis without laboratory prepared or determined reference food adulterant values," Food Chemistry, vol. 148, pp. 289-293, 2014.

[2] M. Woolfe and S. Primrose, "Food forensics: using DNA technology to combat misdescription and fraud," Trends in Biotechnology, vol. 22, no. 5, pp. 222-226, 2004.

[3] N. Rai, D. Banerjee, and R. Bhattacharyya, "Urinary melamine: proposed parameter of melamine adulteration of food," Nutrition, vol. 30, pp. 380-385, 2014.

[4] J. L. Dorne, D. R. Doerge, M. Vandenbroeck et al., "Recent advances in the risk assessment of melamine and cyanuric acid in animal feed," Toxicology and Applied Pharmacology, vol. 270, no. 3, pp. 218-229, 2013.

[5] S. Serranti, D. Cesare, F. Marini, and G. Bonifazi, "Classification of oat and groat kernels using NIR hyperspectral imaging," Talanta, vol. 103, pp. 276-284, 2013.

[6] C. R. Hurburgh, G. R. Rippke, C. Heithoff, S. A. Roussel, and C. L. Hardy, "Detection of genetically modified grains by nearinfrared spectroscopy," in Proceedings of the PITTCON 2000Science for the 21st Century, p. 1431, New Orleans, La, USA, March 2000.

[7] L. Munck, J. Pram Nielsen, B. Møller et al., "Exploring the phenotypic expression of a regulatory proteome-altering gene by spectroscopy and chemometrics," Analytica Chimica Acta, vol. 446, no. 1-2, pp. 171-186, 2001.

[8] M. R. Campbell, J. Sykes, and D. V. Glover, "Classification of single- and double-mutant corn endosperm genotypes by nearinfrared transmittance spectroscopy," Cereal Chemistry, vol. 77, no. 6, pp. 774-778, 2000.

[9] Y. Rui, Y. Luo, K. Haung, W. Wang, and L. Zhang, "Discrimination of transgenic corns using NIR diffuse reflectance spectroscopy and back propagation (BP)," Spectroscopy and Spectral Analysis, vol. 25, pp. 1581-1592, 2005.

[10] R. L. Wehling, D. S. Jackson, and B. R. Hamaker, "Prediction of corn dry-milling quality by near-infrared spectroscopy," Cereal Chemistry, vol. 73, no. 5, pp. 543-546, 1996.

[11] S. R. Delwiche, "Protein content of single kernels of wheat by near-infrared reflectance spectroscopy," Journal of Cereal Science, vol. 27, no. 3, pp. 241-254, 1998. 
[12] M. R. Campbell, S. R. Mannis, H. A. Port, A. M. Zimmerman, and D. V. Glover, "Prediction of starch amylose content versus total grain amylose content in corn by near-infrared transmittance spectroscopy," Cereal Chemistry, vol. 76, no. 4, pp. 552$557,1999$.

[13] S. Kawamura, M. Natsuga, and K. Itoh, "Determination of undried rough rice constituent content using near-infrared transmission spectroscopy," Transactions of the American Society of Agricultural Engineers, vol. 42, no. 3, pp. 813-818, 1999.

[14] N. Berardo, V. Pisacane, P. Battilani, A. Scandolara, A. Pietri, and A. Marocco, "Rapid detection of kernel rots and mycotoxins in maize by near-infrared reflectance spectroscopy," Journal of Agricultural and Food Chemistry, vol. 53, no. 21, pp. 8128-8134, 2005.

[15] D. Özdemir, "Genetic multivariate calibration for near infrared spectroscopic determination of protein, moisture, dry mass, hardness and other residues of wheat," International Journal of Food Science and Technology, vol. 41, no. 2, pp. 12-21, 2006.

[16] H. Huang, H. Yu, H. Xu, and Y. Ying, "Near infrared spectroscopy for on/in-line monitoring of quality in foods and beverages: a review," Journal of Food Engineering, vol. 87, no. 3, pp. 303-313, 2008.

[17] V. K. Gupta, A. Das, and A. Dey, "Universal optimality of block designs with unequal block sizes," Statistics and Probability Letters, vol. 11, no. 2, pp. 177-180, 1991.

[18] A. Savitzky and M. J. E. Golay, "Smoothing and differentiation of data by simplified least squares procedures," Analytical Chemistry, vol. 36, no. 8, pp. 1627-1639, 1964.

[19] R. J. Barnes, M. S. Dhanoa, and S. J. Lister, "Standard normal variate transformation and de-trending of near-infrared diffuse reflectance spectra," Applied Spectroscopy, vol. 43, no. 5, pp. 772777, 1989.

[20] Q. S. Xu and Y. Z. Liang, "Monte Carlo cross validation," Chemometrics and Intelligent Laboratory Systems, vol. 56, no. 1, pp. 1-11, 2001.

[21] L. Xu, P. Shi, Z. Ye, S. Yan, and X. Yu, "Rapid analysis of adulterations in Chinese lotus root powder (LRP) by nearinfrared (NIR) spectroscopy coupled with chemometric class modeling techniques," Food Chemistry, vol. 141, no. 3, pp. 24342439, 2013.

[22] D. M. Haaland and E. V. Thomas, "Partial least-squares methods for spectral analyses. 1. Relation to other quantitative calibration methods and the extraction of qualitative information," Analytical Chemistry, vol. 60, no. 11, pp. 1193-1202, 1988.

[23] D. M. Haaland and E. V. Thomas, "Partial least-squares methods for spectral analyses. 2. Application to simulated and glass spectral data," Analytical Chemistry, vol. 60, no. 11, pp. 12021208, 1988. 

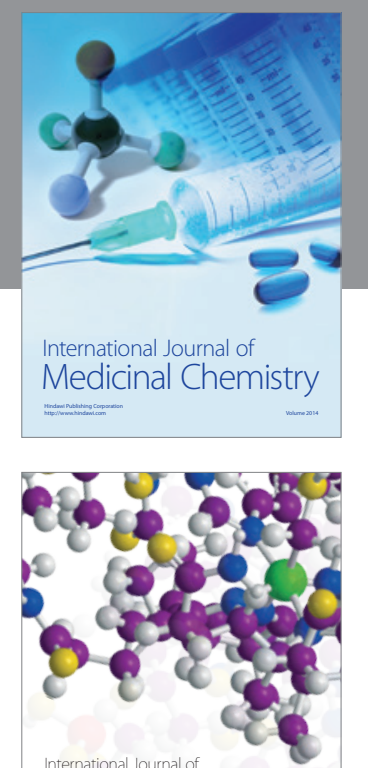

\section{Carbohydrate} Chemistry

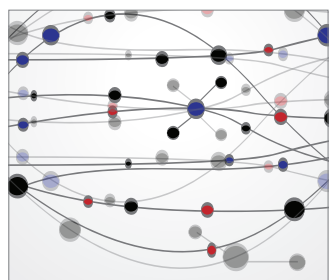

The Scientific World Journal
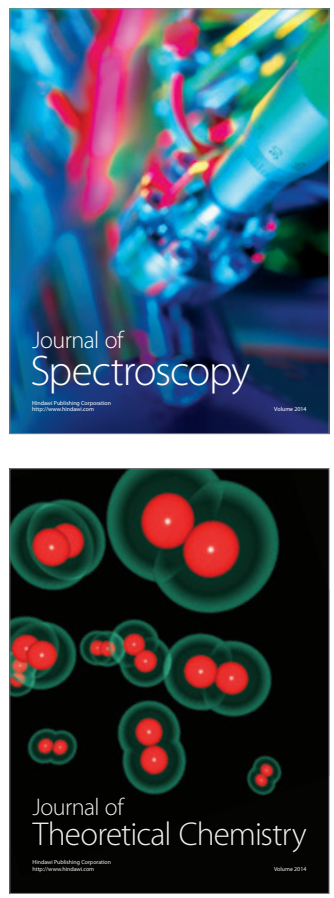
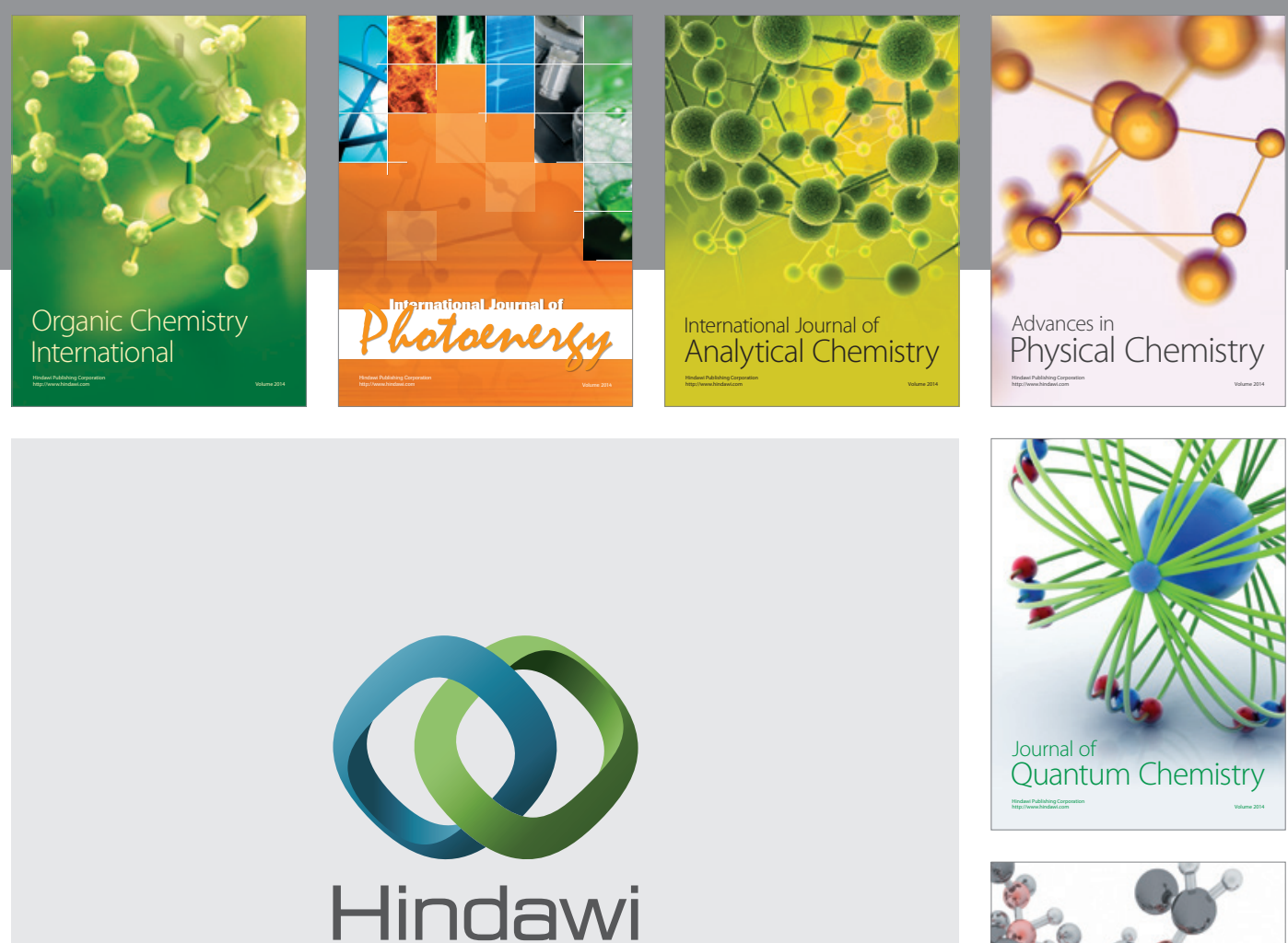

Submit your manuscripts at

http://www.hindawi.com

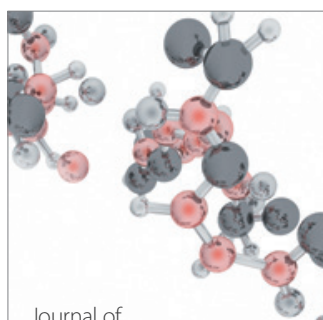

Analytical Methods

in Chemistry

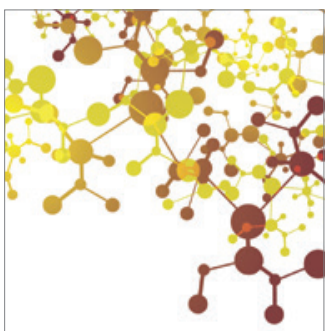

Journal of

Applied Chemistry

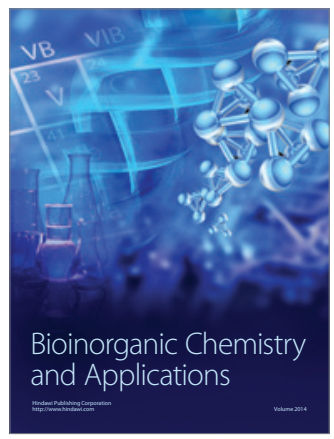

Inorganic Chemistry
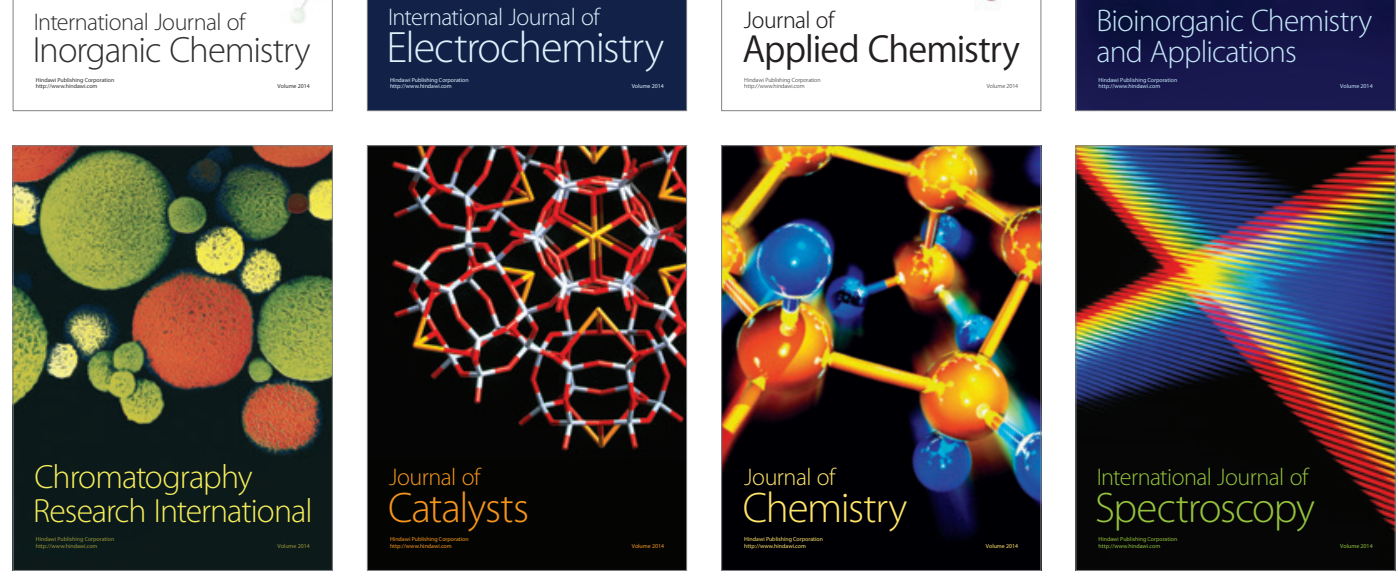